\title{
El Arte del Poder Monárquico: Cuentos Sacados de Cerámica Pintada
}

Dorie Reents-Budet

Ronald L. Bishop

\section{Resumen}

La cerámica clásica maya pintada representa una fuente valiosa de información acerca de la composición física, social, política y psicológica de la corte real maya. Las escenas pintadas de las vasijas proveen detalles de los componentes idealistas y de la naturaleza jerárquica de la corte, proporcionando una oportunidad única para estudiar la vida aristocrática maya y de los cargos monárquicos y sobre los individuos históricos quienes dirigían la sociedad antigua como personalidades únicas en vez de personas sin cara de un pasado distante.

Palabras Clave: Cerámica Maya Clásica, Vida de la Monarquía, Arte Maya.

\section{Abstract}

The painted classic Mayan pottery represents a valuable source of information about the physical, social, political and psychological composition of the Maya royal court. The painted scenes of the vessels provide details of the idealistic components and the hierarchical nature of the court, providing a unique opportunity to study Mayan aristocratic life and monarchical positions and on the historical individuals who ruled the ancient society as unique personalities instead of faceless people from a distant past.

Keywords: Classic Maya Ceramics, Courtly Life, Maya Art.

Dorie Reents-Budet, (doriebudet@frontier.com) Ronald L. Bishop, Museum of Fine ArtsBoston. 


\section{INTRODUCCIÓN}

Los mayas del período Clásico son famosos por su arquitectura sobresaliente y por su escultura monumental en piedra. Produjeron también murales exquisitamente pintados para adornar edificios, de los cuales solamente unos pocos han sobrevivido para proveer evidencia de esta tradición estética (e.g. Bonampak, México [Miller 2013] y San Bartolo, Guatemala [Saturno 2002, 2003a, 2003b]). Sin embargo, cientos de pinturas han sobrevivido en cerámica, habiendo los mayas creado una tradición de pintura altamente sofisticada sobre la superficie curva de vasijas de barro y en tierra sigilada. Éstas son maravillas de la difícil técnica de pintura por engobe en fuego bajo también son obras maestras de composición gráfica y de caligrafía (Reents-Budet et al. 1994, Coe y Kerr 1998).

La cerámica clásica maya pintada representa una fuente valiosa de información acerca de la composición física, social, política y psicológica de la corte real (Reents-Budet 2002a, Miller y Martin 2004). Las escenas pintadas de las vasijas proveen detalles de los componentes idealistas y de la naturaleza jerárquica de la corte, proporcionando una oportunidad única para estudiar la vida aristocrática maya y de los cargos monárquicos y sobre los individuos históricos quienes dirigían la sociedad antigua como personalidades únicas en vez de personas sin cara de un pasado distante.

\section{IMÁGENES DE LA CORTE MONÁRQUICA}

Los pintores mayas crearon imágenes compuestas de la arquitectura y de la gente de la corte real. Versiones de la arquitectura palaciega dan color y vida a los ahora esqueléticos edificios de piedra. Las bandas rojas del borde, arriba y bajo de la composición pictórica, representan las fachadas superiores y la grada superior de la plataforma de los edificios reales. En ocasiones los artistas representan la decoración perdida de estuco-y-pintura, y a veces usan los textos jeroglíficos como elementos arquitectónicos, por ejemplo como las molduras basales y gradas más bajas. También pintan cortinas del palacio y quizá pintaban textiles y tendales para sombra que otrora adornaban los frentes de edificios administrativos o rituales. En algunos casos, los diseños adornando a los textiles podrían haber indicado las funciones o papeles oficiales de aquellos edificios. Los pintores mayas de vez en cuando representaban los típicos techos de peine de las estructuras de la élite del período Clásico. Algunas de las vasijas más estimulantes son aquellas cuya forma y decoración transforman la vasija en un edificio, incluyendo el techo de paja que también se refiere al petate tejido de la autoridad real. 


\section{MIEMBROS DE LACORTE}

Las escenas de la corte real son retratos formales, pues los artistas nunca pintaron el lado doméstico de la vida en el palacio ni las preparaciones fuera de escenario. La representación ocasional de ponerse la vestimenta real o vestimenta de actuación no es una imagen privada sino un componente público formal del evento cortesano.

La corte maya era un lugar bullicioso con muchos oficiales atendiendo deberes específicos y participando en los quehaceres del estado. Vemos al gobernante recibiendo a extranjeros de otros cortes reales incluyendo presentadores de regalos, historiadores del corte y escribanos para contar la historia pasada y grabar los eventos del momento (el aj k'uhun y el aj ts'ib; ver también a Stuart 1987 en cuanto a una discusión sobre pintores cortesanos de libros). Los acarreadores de literas son trompeteros quienes anuncian las llegadas y las presentaciones de regalos, en el caso de la algunas vasijas dos bolsas de valiosas conchas spondylus. Los actores aristocráticos establecen de nuevo historias épicas y eventos históricos demostrando los logros del rey, demostrando sus ancestros y los dioses, enfatizando así la base divina del gobierno.

Figuras cortesanas están paradas detrás del trono y presencian el evento. Algunos pueden ser la contraparte del "gran maestro de brindis" de la corte K'iché del siglo $\mathrm{XVI}$, quien era maestro de ceremonias como también observador a favor del rey (Tedlock 1996:322). Otra figura que siempre está de pie muy cercana al trono y a menudo junto a una percha de bienes valiosos, trayendo a la mente al cortesano K'iché ajpop k'amja del siglo XIV, o 'custodio del petate de la Casa de Recepción'. Dennis Tedlock (1996:315) anota que entre las funciones de esta persona estaba la de recolección de tributo. Los retratos de este individuo durante el período clásico a menudo lo demuestran con un cigarrillo o antorcha, inundando el palacio con luz y perfumando la atmósfera de la corte (Reents-Budet 2001a:213-218).

Las mujeres eran miembros importantes de la corte monárquica. Eran las amas apropiadas, asegurando un evento memorable, enfatizándolo con una fiesta pomposa, siendo una de las comidas requisito las bebidas de chocolate, perfectamente preparadas para crear una bebida espumosa de buen sabor y de buen aroma. Las mujeres eran también actores, artistas y proveedoras de poder imperial como reinas y madres. Así como en otras partes del mundo, casamientos 
monárquicos se arreglaban para servir a fines políticos. Por ejemplo como un extraño, K'inich Yax K'uk' Mo' se casó con una copaneca de la élite para afijar su posición política y establecer una dinastía nueva en el Valle de Copán (Sharer, Fash, et al.1999; también ver a Marcus 1992).

Escenas en barro preservan momentos clave en la historia aristocrática, proporcionando los nombres de muchos participantes quienes de otra manera son invisibles en el registro arqueológico. Por ejemplo, una vasija representa los ritos de entronización de Yajaw Te' K'inich quien gobernó la comunidad lk' (el moderno Motul de San José en las tierras bajas del Petén de Guatemala) durante mediados del siglo VIII, siendo su accesión en este caso atestiguada por el gobernante Lamaw Ek'. Los ritos de designación del heredero destacan la presentación formal del joven príncipe y su actuación ritual para los señores reunidos. Las reuniones diplomáticas trataban sobre conflictos o alianzas, apareciendo en esta vasija el emisario del gobernante de Calakmul, nombrado en los textos jeroglíficos como K'ak' Hiix, presentando un regalo de su jefe Yich'aak K'ak' a Jasaw Chan K'awiil, gobernante divino de Tikal, ciudad enemiga principal de Calakmul. El regalo se compone de un bulto de 12,000 semillas de cacao (3-pik, como aparece en el bulto; ver a Stephen Houston en Miller 1997), tela fina y plumas de quetzal (registradas en los dos últimos glifos en la columna vertical de jeroglíficos como yilaj chanlajun pat "él vio 14 tributo") (Stan Guenter 2006: comunicación personal). El evento tuvo lugar en el sitio de Topoxte', un centro insular en el Lago Petén-Itzá, en 9.12.19.10.0 4-Ajaw 13-Keh, es decir, el 7 de octubre del año 691 d.C. Otras vasijas demuestran las responsabilidades rituales del gobernante como los ritos en búsqueda de una visión, durante los cuales acumulaban poder sagrado para influir en los quehaceres humanos, estas actuaciones de transformación o de impersonificación afirmaban públicamente las habilidades especiales del gobernante. Otras escenas demuestran la hazaña económica del gobernante en la forma de regalos de aliados políticos y tributo de territorios subyugados.

\section{FUNCIÓN DE LA VASIJA}

¿Por qué eventos históricos tan críticamente importantes se conservarían en vasijas pequeñas de barro y no en obras monumentales de piedra de mayor impacto visual y psicológico? La respuesta se encuentra en las funciones de la vasija durante las fiestas aristocráticas que a menudo aparecen en cerámica realizándose dentro de complejos palaciegos como la Acrópolis Sur de Copán (ver Figuras 1, 9; Reents- 
Budet 2000b). Las fiestas acompañaban eventos importantes como la victoria en la guerra, presentación de tributo, llegada al trono, visitas diplomáticas, negociaciones de casamiento, eventos de obligaciones socio-políticas, re-actuaciones épicas realizadas en las anchas escalinatas de un edificio y los rituales relativos a la ideología de gobierno y a la veneración de ancestros reales (Grube 1992, Pohl 1999, Reents-Budet 2000b). Los nombres de los participantes a menudo aparecen en los textos cortos jeroglíficos cerca de ellos y el texto primario en la parte superior de la vasija incluye los títulos elitistas y el nombre del propietario o patrón de la vasija (Reents-Budet et al. 1994:106-163). Esta persona es típicamente la figura principal en la escena, rodeada de vasijas llenas de comidas como tamales cubiertos con salsas sabrosas, atole (pozole), bebidas de chocolate y bebidas fermentadas como balché (Houston, Stuart y Taube 1989).

Los contenidos de las vasijas se confirman por medio de los textos jeroglíficos en los bordes que dan el nombre del tipo de vasija (e.g. uk' "taza para beber", lak' "plato") y sus contenidos (ver a MacLeod, en Reents-Budet et al. 1994:127133). Por ejemplo, los platos contenían tamales (u lak' ti wah, "su (de él o de ella) plato para tamales") y fuentes contenían atole ( $y$-uk'ib ta ul, "su (de él o de ella) vasija para tomar atole"; Taube 1989). Los vasos cilíndricos contenían bebidas de chocolate ( $y$-uk'ib ta tsih te'el kakaw, "su (de él o de ella) vasija de tomar cacao fresco"; Reents-Budet et al. 1994:115-120). El chocolate espumoso (om kakaw) se valoraba altamente porque la espuma contiene la mayoría del aroma y por ende el mayor sabor. El cacao se valoraba no sólo por su sabor y los efectos positivos en el cuerpo y en la mente sino también porque el cacao es un indicador directo de la riqueza y estatus del anfitrión porque es difícil cultivar y por lo tanto era escaso y costoso de obtener (ver a Richard Blanton et al. 1996).

Estas escenas pintadas del período Clásico e informaciones similares escritas en el siglo XVI subrayan la importancia de las fiestas como parte integral de los rituales mayas del poder monárquico. Los arqueólogos con frecuencia encuentran grandes depósitos de basura junto a edificios palaciegos que seguramente son los restos de fiestas (Brown 2001, Reents-Budet 2000b, Reents-Budet et al. 2000). Por ejemplo, el basurero que se encontró fuera del Popol Nah en Copán (Estructura 22A) contenía cerámica, huesos de animales comestibles como venado y herramienta de piedra que Kazuo Aoyama ha determinado se utilizaban para cortar carne (Aoyama 2001). También contienen cerámica policromada, cuya mayoría está decorada con estilos artísticos locales (ver a Bill 1997). 
El montaje de y la participación en una fiesta crea redes de relación y obligaciones de reciprocidad, uniendo a los participantes (Earle 1997; Monaghan 1990; Pohl 1994, 1999). Así como en otras partes del mundo donde los festejos están en el centro de la vida social y de la práctica política, la fiesta incluye no sólo el consumo de grandes cantidades de excelente comida pero también la distribución de muy bien hechos regalos (Junker 2001). En la Yucatán del siglo XVI, el Obispo Diego de Landa escribe que tela finamente tejida era un regalo importante durante las fiestas (Tozzer 1941). Igualmente durante el período Clásico, el tributo y la comodidad de regalo que más frecuentemente se representan en las escenas pintadas es tela tejida (Reents-Budet 2007). La tradición de tela artísticamente tejida y su importancia para las relaciones sociales sobrevive hoy día entre muchos grupos mayas en Guatemala y México (Morris 1987). Otros regalos de fiestas que se ilustran en cerámica del período Clásico incluye exóticos como plumas de quetzal, conchas spondylus, adornos de jadeíta y cerámica pintada (para datos comparativos entre los aztecas del siglo XVI, ver a Brumfiel 1987, Burgoa 1989; para datos comparativos entre los mixtecas ver a Byland y Pohl 1994).

De manera que cerámica exquisitamente pintada funcionaba no sólo como un grupo de servicio para estatus alto durante fiestas sino también como "moneda social", es decir, regalos influyentes que se presentaban a los participantes (Junker 2001:274-276, Price 1989). Estas vasijas eran un símbolo público de la riqueza y del poder del anfitrión porque la imaginería y la habilidad artística reflejan el vigor y la autoridad del él, la dinámica de su corte, el origen divino del poder monárquico y su (de él o de ella) habilidad en dirigir labor humana altamente especializada.

El fin último de la fiesta era asegurar alianzas sociales y políticas como parte de un sistema basado en una estrategia de redes con otros gobernantes en combinación con estrategias corporativas manipulando relaciones familiares y sociales (ver a Blanton et al.1996). El anfitrión servía grandes cantidades de comida y distribuía entre los huéspedes bienes básicos y artículos finamente elaborados por medio de los cuales se forjaban lazos de obligación entre el anfitrión y el huésped. La distribución de comodidades y de artículos valiosos proclamaba el poder del anfitrión, siendo su regalo un estímulo potente para atraer miembros a su red de alianza. La riqueza es poder porque actúa como una moneda dinámica que sostiene relaciones sociales y alianzas políticas. 
De la misma manera que en otras partes del mundo, cuando el arte funciona en el ámbito socio-político, requiere de "aditivos de prestigio" para transformar el objeto cotidiano en un regalo de influencia y de poder (Price 1989, Reents-Budet et al. 1994:72-105). Entre los aditivos de prestigio están la excelencia artística, estilo único, imágenes representando a gente importante e ideologías de poder, el nombre del apadrinador y la firma del artista. Todas éstas son características primarias de la cerámica pintada del Clásico Tardío maya. La importancia del artista para la corte monárquica se enfatiza por medio de la identidad del hombre quien pintó dos obras maestras. Firmó la vasija blanco y negro con su nombre personal y proporciona una lista de sus padres-la Señora real de Yaxhá y el Señor K'ak' Ukala Chan Chaak, el gobernante de Naranjo a mediados del siglo VIII (755-c. 780; Stuart 1987).

El estilo de la pintura también puede proveer datos útiles para re-tejer los hilos antiguos de la tapicería complicada de las conexiones sociales y del poder político durante los tiempos del Clásico Tardío. Para hacer esto, no obstante, los estilos deben identificarse y conectarse con sus lugares de producción y de uso. Hacia este objetivo se creó durante la década de los años 1970 el Proyecto de Cerámica Maya en los Laboratorios Nacionales de Brookhaven con el apoyo del Museo de Bellas Artes Boston. Ahora el proyecto se ha movido al Museo Nacional de Historia Natural, Institución Smithsoniana, Washington, DC.

El Proyecto de Cerámica Maya utiliza la técnica analítica altamente sensitiva de análisis instrumental de activación neutrónica para caracterizar químicamente las pastas de tiestos de cerámica y de vasijas completas que nos permiten descubrir donde se hizo una vasija (Bishop, Rands y Holley 1982; Glascock 1992). Del fondo de una vasija, se remueve una mínima cantidad (100 miligramos) de la pasta cerámica y el área se restaura rellenando y pintando con materiales apropiados de conservación. La muestra se bombardea con neutrones dentro de un reactor nuclear en el Instituto Nacional de Estándares y Tecnología en Washington, DC. El resultado final es una lista de vestigios de elementos, en partes por millón, que contiene la pasta cerámica de la vasija. Esta lista sirve como una 'huella dactilar química' de la mezcla única de barros y desgrasantes, formulados por el ceramista antiguo. Por medio de la comparación del patrón químico de la muestra con aquellos de los tiestos excavados en un sitio como también de otras localidades, se puede determinar el lugar de manufactura (taller de origen). De esta manera, una vasija de cerámica y su estilo de pintura se puede atribuir a una región geográfica 0 aun a un sitio específico (Rands y Bishop 1980). 
Conociendo el origen y el lugar final de descanso de una vasija provee una indicación directa de la interacción social que quizá no se haya preservado en ninguna otra forma. Por ejemplo, Altun Ha, Belice fue una ciudad influyente durante los siglos VI y VII (Pendergast 1979). Es famosa por los adornos espectaculares de jadeíta que se enterraron con sus gobernantes, incluyendo la pieza única más grande de jadeíta, trabajada por los mayas del Clásico y una joya grande de pectoral que representa el retrato de un gobernante de Altun Ha y un registro jeroglíico de su ascensión al trono. Interesantemente, su estilo y la calidad de su técnica fuertemente se asemejan a las joyas de jadeíta que se han encontrado en las tumbas reales de Copán.

Los basureros palaciegos en Altun Ha contienen grandes cantidades de cerámica policromada, muchas pintadas en un estilo único y cuya química de pasta indica producción local (Figura 18a; Reents-Budet et al. 1994:198-203). Una vasija pintada en este estilo se excavó de una tumba de un miembro de la élite en la Acrópolis Sur en Copán (Figura 18b; ver también a Longyear 1952:40, Fig. 108a), cuyo estilo y composición química indican que su origen fue Altun $\mathrm{Ha}$. La jadeíta y los datos sobre la cerámica importada en ambos sitios juntos indican relaciones sociales, políticas y/o económicas entre Copán y Altun Ha durante el medio del período Clásico.

El Instituto Hondureño de Antropología e Historia permitió sacar muestras químicas de cerámica de las tumbas del Clásico Temprano de Copán, incluyendo la Tumba Hunal, considerada ser la del fundador de la dinastía K'inich Yax K'uk' Mo' (Bell, et al. 2004, Fash 1991:84; Sharer 1997; Sharer y Sedat 1994; ver también a Schele 1986; Stuart y Schele 1986). El objetivo era entender mejor las implicaciones sociales y políticas de estas vasijas importantes para enfrentar preguntas sobre el origen de la familia real y especialmente sobre el fundador de la dinastía.

Las vasijas de la Tumba Hunal incluyen una mezcla de cerámica hecha localmente (12 vasijas), una de la vecindad de Quiriguá y una vasija extraordinaria efigie de venado desde el área de Kaminaljuyú (la Ciudad actual de Guatemala) (Figura 19; Reents-Budet y Bishop 2004:169-174). Dos jarros trípodes se hicieron en 0 cerca de Tikal (ver Bell, et al 2004: Plate 5b). La química de su pasta y sus estilos de decoración son similares a cuatro tiestos policromos que se excavaron en los 1950s en el complejo residencial real de Copán 10L-2, implicando conexiones relativamente fuertes con el área de Tikal durante algún tiempo (Reents-Budet et al.1993; ver también a Culbert 1993). Por fin, dos cuencos con base de anillo y un 
jarro alto se hicieron en talleres en las tierras altas centrales de México (ReentsBudet y Bishop 2004:Figura 9.8; ver también Rattray y Harbottle 1992).

En su totalidad, la cerámica de la Tumba Hunal representa un conjunto "internacional" que seguramente refleja las relaciones socio-históricas del ocupante real de la tumba. Las vasijas no apoyan la conjetura anterior de que K'inich Yax K'uk' Mo' tenía fuertes lazos con Kaminaljuyú (Longyear 1952; Reents-Budet y Culbert 1999). En vez de esto, sus lazos estaban más igualitariamente distribuidos entre un número de regiones, en particular las tierras altas centrales de México y el área de Tikal. Las inscripciones jeroglíficas de Copán declaran que K'inich Yax K'uk' Mo' llegó de otro lugar aunque no especifican de dónde (Martin y Grube 2000:192-193; Stuart 2000). Se ha propuesto un origen en las tierras altas centrales de México, basándose en los rasgos de su vestimenta real que recuerdan los de Teotihuacan (Fash 1991:23-24; también ver a Kubler 1967; Langley 1992; Pasztory 1976). Pero, a pesar de que tres vasijas de la tumba se hicieron en las tierras altas de México, la mayoría se hizo localmente y las conexiones cerámicas con el área de Tikal son igualmente fuertes, si no dominantes. Esta hipótesis coincide con el análisis por Jane Buikstra de la proporción de isotopo en los restos esqueléticos de K'inich Yax K'uk' Mo', que indica que él alcanzó madurez física en el área de Tikal (Buikstra et al. 2000).

Un segundo ejemplo de Copán es la Tumba Margarita, la que se cree ser la tumba de la esposa de K'inich Yax K'uk' Mo' (Bell, Sharer et al. 2004:141; Sharer, Traxler et al. 1999). El nombre de esta mujer no se registró en ningún monumento conocido, pero su tumba contiene gran cantidad de información personal. De sus huesos, Jane Buikstra determinó que era una mujer local de buena salud y buena estatura (Buikstra et al. 2000; Buikstra et al. 2004:201). Las composiciones de la pasta de las vasijas de la tumba corroboran su origen local y también indican relaciones "extranjeras" con Kaminaljuyú en las tierras altas de Guatemala, el área de Tikal en el Petén central y las tierras altas de México cerca de Teotihuacan (Figura 20; Reents-Budet et al. 2004:174-181). Junto con las vasijas de la Tumba Hunal, los datos cerámicos soporte la interpretación que el Clásico Temprano de Copán emerge como una comunidad importante cuyo fundador dinástico vino más recientemente de la región de Tikal y consolidó su poder, en parte, casándose con una familia aristocrática local. Sus conexiones internacionales le proveyeron la influencia, la riqueza y el poder para consolidar una dinastía real autoritaria.

Las vasijas pintadas del Clásico maya también pueden darnos una idea sobre la personalidad del personaje real, más allá de los retratos de fórmula en piedra de la persona oficial. Uno de los gobernantes más reconocidos es Yajaw Te' K'inich quien 
gobernó la comunidad Ik' durante la parte media del siglo VIII (Figura 21; ReentsBudet et al. 1994:172-179; Reents-Budet, Foias et al. 2007). Su artista favorecido reprodujo las características físicas únicas de Yajaw Te' K'inich. Aquí vemos debajo de la superficie de los monárquicos tradicionales y encontramos un padrino quien apreciaba la fineza de la línea, la delicadez de baños de color y los acentos visuales de matices poco comunes. Yajaw Te' K'inich prefería un retrato de realidad física a uno de representación estilizada dentro de los cánones establecidos de belleza maya, basados en el dios del Maíz (Fields 1986, Fields et al. 2005). El maestro artista pintó a Yajaw Te' K'inich con todas sus ostentaciones de cuerpo grande y de presencia psicológica.

Yajaw Te' K'inich puede describirse como un aficionado de pintura fina, conspicuo en sus necesidades materiales, con confianza en sí y jactancioso de sus logros. Su apadrinamiento de muchas fiestas con la distribución requerida de regalos, incluyendo vasijas de fiesta pintadas, se da a entender indirectamente por la existencia de más de trece vasijas, mostrando al gobernante y producidas tanto por su pintor principal como por artistas menores, vasijas todas hechas en el área de Motul de San José (Reents-Budet et al. 2007, 2012). Podemos conjeturar que Yajaw Te' K'inich fue un maestro de los procesos socio-políticos y económicos, acumulando riqueza y alianzas para incrementar su posición social y política durante mediados del volátil siglo VIII, en la región del Petén central.

En contraste tenemos a Jasaw Chan K'awiil (reinó del 682 al 734 d.C.), el famoso gobernante de Tikal quien controlaba a este superpoder inmediatamente antes de la llegada al poder de Yajaw Te' K'inich de la comunidad lk' ubicado al sur de Tikal (Harrison 1999:125-146; Martin y Grube 2000: 44-47). Entre sus importantísimos logros fue la derrota de Calakmul, el otro superpoder del período Clásico Tardio, y continuó hasta controlar el Petén central, incluyendo la comunidad de lk'. Jasaw Chan K'awiil fue la fuerza vital detrás de la revitalización de Tikal, después de siglos de decadencia y de liderazgo sin inspiración. Él apoyó el período de mayor construcción en Tikal, incluyendo los Templos I y II y agregados mayores al palacio real. Su tumba contenía obras fenomenales de arte, incluyendo una vasija con incrustaciones de jade, sosteniendo cada placa de jade con un pequeño taco de jade y la agarradera trabajada para representar a Jasaw Chan K'awiil como el dios del Maíz (Harrison 1999:145). Los exquisitamente incisos huesos de jaguar que se recuperaron de su tumba presentan al rey como el dios del Maíz hundiéndose en el Inframundo al momento de su muerte.

Sin embargo, una comparación de estas elegantes obras de arte con las vasijas que se encontraron en su tumba resalta su sorprendente pobre calidad (ver Culbert 
1993:Figuras 69-75). Las vasijas inusitadamente gruesas se han hendido debido a problemas técnicos, y la calidad de la pintura está entre la peor que se ha encontrado entre la cerámica de la realeza del período Clásico. Aun más curioso y que contradice a estas vasijas pobremente pintadas, es la réplica en cerámica de una olla de pintura, típicamente hecho de una concha marina, que se encontró entre las ofrendas del entierro del rey (Culbert 1993: Figura 65a; Reents-Budet et al. 1994: Figura 2:10).

Cuando se compara con la cerámica de Yajaw Te' K'inich, la de la tumba de Jasaw Chan K'awiil nos lleva a la especulación que quizá él esquivó la fineza de las fiestas como una afirmación de exclusividad. Quizá su poder era tal que no necesitaba fiestas de política como mecanismo de autoridad y de poder. 0 quizá no le gustaba o no confiaba en multitudes de nobles hambrientos de poder o quizá simplemente no le importaba o no podía discernir la calidad artística. La olla de pintura que se extrajo de la tumba trae la especulación que quizá se creía un gran artista y pintaba sus propias vasijas (Coggins 1975). Sin importar la interpretación correcta, percibimos a un gobernante de supremo poder cuya cerámica funeraria sugiere que no estaba participando de los típicos ornamentos del sistema de fiestas políticamente cargado.

En resumen, la cerámica del Clásico maya incluye una de las tradiciones fenomenales mundiales de cerámica, reconocida por su complejidad pictórica, sus logros calígrafos y literarios y por su diversidad artística que todos juntos dan testimonio a la creatividad insuperable de los pintores mayas. No obstante, estas vasijas no son simplemente objetos poderosos de belleza y de admiración, sino ventanas primarias hacia los movimientos internos del poder de la corte y de las personalidades individuales quienes guiaban la sociedad antigua maya.

\section{BIBLIOGRAFIA}

- Aoyama, Kazuo (2001) Classic Maya Craft Specialization and Elite Domestic Activities: Lithic Evidence from Aguateca, Guatemala. Trabajo presentado en la 66 Reunión Anual de la Society for American Archaeology. New Orleans.

- Bell, Ellen, R. Sharer, L. Traxler, D. Sedat, C. Carrelli, y L. Grant, (2004). Tombs and Burials in the Early Classic Acropolia at Copan. En Understanding Early Classic Copan, editado por E. Bell, M. Canuto, y R. Sharer, pp. 132-157. University of Pennsylvania Press. 
- Bill, Cassandra, (1997) Patterns of Variation and Change in Dynastic Period Ceramics and Ceramic Production at Copán, Honduras. Disertación Doctoral, Departmento de Antropología, Tulane University. New Orleans.

- Bishop, Ronald L., R. L. Rands, y G. R. Holley, (1982). $\quad$ C e r a $m$ i c Compositional Analysis in Archaeological Perspective. En Advances in Archaeological Method and Theory, Vol. 5, editado por M. B. Schiffer, pp. 275331. New York: Academic Press.

- Blanton, Richard, G. Feinman, S. Kowalewski, y P. Peregrine, (1996) A DualProcessual Theory for the Evolution of Mesoamerican Civilization. En Current Anthropology 37(1):1-14.

- Brown, Linda A., (2001). Feasting on the Periphery: The Production of Ritual Feasting and Village Festivals at the Cerén Site, El Salvador. En Feasts: Archaeological and Ethnographic Perspectives on Food, Politics, and Power, editado por M. Dietler y B. Hayden, pp. 368-390. Washington, D.C.: Smithsonian Institution Press.

- Brumfiel, Elizabeth, (1987). Elite and Utilitarian Crafts in the Aztec State. En Specialization, Exchange and Complex Societies, editado por E. Brumfiel y T. Earle, pp. 102-118. Cambridge: Cambridge University Press.

- Buikstra, Jane, D. Price, J. Burton, y L. Wright, (2000). The Early Classic Royal Burials at Copán: A Bioarchaeological Perspective. Trabajo presentado en la asamblea titulada Understanding Early Classic Copán. Reunión Annual de la Society for American Archaeology, April 5-9, 2000. Philadelphia.

- Buikstra, Jane, D. Price, L. Wright, y J. Burton, (2004). Tombs from the Copan Acropolis: A Life History Approach. En Understanding Early Classic Copan, editado por E. Bell, M. Canuto y R. Sharer, pp. 191-214. University of Pennsylvania Press.

- Burgoa, Fray Francisco de 1989[1674] Geográfica Descripción. Vol. 1. México: Editorial Porrúa.

- Byland, Bruce E., y J. Pohl, (1994). In the Realm of 8 Deer: The Archaeology of the Mixtec Codices. Norman: University of Oklahoma Press.

- Coe, Michael, y J. Kerr, (1998). Art of the Maya Scribe. London y New York: Thames and Hudson, Ltd. 
- Coggins, Clemency, (1975). Painting and Drawing Styles at Tikal. Disertación Doctoral. University Microfilms International, Ann Arbor.

- Culbert, T. Patrick, (1993). The Ceramics of Tikal: Vessels from the Burials, Caches and Problematical Deposits. University Museum Monograph 81. Editores de la Serie William R. Coe y William A. Haviland. Philadelphia: The University Museum, University of Pennsylvania.

- Earle, Timothy, (1977). Reappraisal of Redistribution: Complex Hawaiian Chiefdoms. En Exchange Systems in Prehistory, editado por T. Earle, pp. 213229. New York: Academic Press.

- Fash, William L., (1991). Scribes, Warriors, and Kings: The City of Copán and the Ancient Maya. London y New York: Thames y Hudson.

- Fields, Virginia M. (1986). The Origins of Divine Kingship among the Lowland Classic Maya. Disertación Doctoral. Departmento de Estudios Latino Americanos.

- La Universidad de Tejas en Austin. (1991). The Iconographic Heritage of the 'Jester God'. En Sixth Palenque Round Table, 1986, editado por Virginia M. Fields, pp. 167-174. Norman: University of Oklahoma Press.

- Fields, Virginia, y D. Reents-Budet, (2005). Lords of Creation: The Origins of Sacred Maya Kingship. Los Angeles County Museum of Art, y Scala Publishers, Ltd. Los Angeles y London.

- Glascock, Michael D., (1992). Characterization of Archaeological Ceramics at MURR by Neutron Activation Analysis and Multivariate Statistics. En Chemical Characterization of Ceramic Pastes in Archaeology, editado por H. Neff, pp. 11-26. Monographs in World Archaeology, No. 7. Madison: Prehistory Press.

- Grube, Nikolai, (1992). Classic Maya Dance: Evidence from hieroglyphs and iconography. Ancient Mesoamerica 3:201-218.

- Harrison, Peter, (1999). The Lords of Tikal: Rulers of an Ancient Maya City. Thames y Hudson, London y New York.

- Houston, Stephen, D. Stuart, y K. A. Taube, (1989). Folk Classification of Classic Maya Pottery. American Anthropologist 91(3):720-726. Washington, D.C. 
- Junker, Laura Lee, (2001). The Evolution of Ritual Feasting Systems in Prehispanic Philippine Chiefdoms. En Feasts: Archaeological and Ethnographic Perspectives on Food, Politics, and Power, editado por M. Dietler y B. Hayden, pp. 267-310. Smithsonian Institution Press, Washington, D.C.

- Kubler, George, (1967). The Iconography of the Art of Teotihuacán. Studies in Precolumbian Art and Archaeology, No. 4. Washington, D.C.: Dumbarton Oaks, The Trustees of Harvard University.

- Langley, James C.,(1992). Teotihuacán Sign Clusters: Emblem or Articulation? En Art, Ideology, and the City of Teotihuacán, editado por J. C. Berlo, pp. 247280. Washington, D.C.: Dumbarton Oaks, The Trustees of Harvard University.

- Longyear, J. M. (1952). Copán Ceramics. A Study of Southeastern Maya Pottery. Carnegie Institution of Washington Publication 597. Washington, D.C.

- Marcus, Joyce, (1992). Mesoamerican Writing Systems: Propaganda, Myth, and History in Four Ancient Civilizations. Princeton, NJ: Princeton University Press.

- Martin, Simon, y N. Grube, (2000). Chronicle of the Maya Kings and Queens. London y New York: Thames y Hudson.

- Miller, Mary Ellen, (1997). Imaging Maya Art. Archaeology 50(3):34-40. New York: Archaeological Institute of America.

- Miller, Mary Ellen, y C. Brittenham, (2013). The Spectacle of the Late Maya Court: reflections on the murals of Bonampak. Instituto $\quad \mathrm{N}$ a $\mathrm{c}$ i o $\mathrm{n}$ a I de Antropología e Historia. Consejo Nacional para la Cultura y las Artes. University of Texas Press, Austin.

- Miller, Mary Ellen, y S. Martin, (2004). Courtly Art of the Ancient Maya. Fine Arts Museums of San Francisco. New York: Thames and Hudson Inc.

- Monaghan, John, (1990). Reciprocity, Redistribution, and the Transaction of Value in the Mesoamerican Fiesta. American Ethnologist 17:758-774. Washington, D.C.: American Anthropological Association.

- Morris, Walter F., Jr., (1987). Living Maya. New York: Harry N. Abrams, Inc. 
- Neff, Hector, (2000). Neutron Activation Analysis for Provenance Determination in Archaeology. En Modern Analytical Methods in Art and Archaeology, editado por E. Ciliberto y G. Spoto, pp 81-134. Chemical Analysis, editior general J. D. Winefordner, Vol. 155. New York: John Wiley y Sons.

- Pasztory, Esther, (1976). The Murals of Tepantitla, Teotihuacán. New York: Garland Press.

- Pendergast, David, (1979). Excavations at Altun Ha, Belize, 1964-1970. Toronto: The Royal Ontario Museum.

- Pohl, John, (1994) The Politics of Symbolism in the Mixtec Codices. Vanderbilt University Publications in Anthropology. Nashville: Vanderbilt University.

- Pohl, John, (1999). The Lintel Paintings of Mitla and the Function of the Mitla Palaces. En Mesoamerican Architecture as a Cultural Symbol, editado por J. Kowalski. Oxford y New York: Oxford University Press.

- Price, Sally, (1989). Primitive Art in Civilized Places. University of Chicago Press. Chicago.

- Rands, R. L., y R. L. Bishop, (1980). Resource Procurement Zones and Patterns of Ceramic Exchange in the Palenque Region, Mexico. En Models and Methods in Regional Exchange, editado por R. E. Fry, pp. 19-46. SAA Papers No. 1. Washington, D.C.: The Society for American Archaeology.

- Rattray, Evelyn C., y G. Harbottle, (1992). Neutron Activation Analysis and Numerical Taxonomy of Thin Orange Ceramics from the Manufacturing Sites of Rio Carnero, Puebla, Mexico. En Chemical Characterization of Ceramic Pastes in Archaeology, editado por H. Neff, pp. 221-232, Monographs in World Archaeology, No. 7. New York: Prehistory Press.

- Reents-Budet, Dorie, (2007). Power Material in Ancient Mesoamerica: The Roles of Cloth among the Classic Maya. En Wrapping Traditions in Ancient Mesoamerica, editado por J. Guernsey y F.K. Reilly, pp. 105-126. The Center for Ancient American Studies, Washington, DC y Ashville, NC.

- Reents-Budet, Dorie, (2000a). Classic Maya Conceptualizations of the Royal Court: An Analysis of Palace Court Renderings on the Pictorial Ceramics. En Royal Courts of the Ancient Maya, editado por Stephen Houston y Takeshi Inomata, pp. 195-236. Boulder: Westview Press. 
- Reents-Budet, Dorie, (2000b). Feasting Among the Classic Maya: Evidence from Pictorial Ceramics. En The Maya Vase Book (Vol. VI), editado por J. Kerr, pp. 1022-1038. New York: Kerr Associates.

- Reents-Budet, Dorie, J. Ball, R. L. Bishop, V. Fields, y B. MacLeod, (1994).

Painting the Maya Universe: Royal Ceramics of the Classic Period. Durham, NC y London: Duke University Press.

- Reents-Budet, Dorie, E. Bell, L. Traxler, y R. Bishop, (2004). Early Classic Ceramic Offerings at Copán: A Comparison of the Hunal, Margarita, and SubJaguar Tombs. En Understanding Early Classic Copan, editado por E. Bell, M. Canuto y R. Sharer, pp. 159-190. University of Pennsylvania Museum of Archaeology and Anthropology. Philadelphia.

- Reents-Budet, Dorie,A. Foias, R. L. Bishop, y J. Blackman, (2007). "Interacciones Políticas y el Sitio Ik' (Motul de San José): Datos de la Cerámica." En XX Simposio (2006) de Investigaciones Arqueológicas en Guatemala, Museo Nacional de Antropología E Etnología, editado por J.P. Laporte, B. Arroyo, y H. Mejía, pp. 11411161. Guatemala: Ministerio de Cultura Y Deportes, IDAEH, Asociación Tikal, y Fundación Arqueológico del Nuevo Mundo.

- Reents-Budet, Dorie, R. L. Bishop, J. Blackman, y S. Guenter, (2012). Identity and Interaction: ceramic styles and social history of the Ik' polity, Guatemala. En Motul de San José: Politics, History, and Economy in a Classic Maya Polity, editado por Antonia Foias y K. Emery, pp. 67-93. Gainesville, FL: University of Florida Press.

- Reents-Budet, Dorie, R. L. Bishop, y B. MacLeod, (1993). Acercamiento Integrado a La Cerámica Pintada Clásica Maya. En VI Simposio de Investigaciones Arqueologícas en Guatemala, 1992. Museo Nacional de Arqueología Y Etnología. Guatemala City: Ministerio de Cultura y Deportes, Instituto de Antropología e Historia y Asociación Tikal.

- Reents-Budet, Dorie, J. Ball, J. Taschek, y R. Bishop. (2000). Out of the Palace Dumps: Ceramic Production and Use at Buenavista del Cayo, Belize. Ancient Mesoamerica 11(1), pp. 99-121.

- Reents-Budet, Dorie, y T. P. Culbert, (1999). Las Ofrendas del período Clásico Temprano de Tikal y Kaminaljuyú: Relaciones Regionales y 'Internacionales.' Trabajo presentado en el XIII Simposio de Arqueología y Etnología de 
Guatemala. Museo Nacional de Arqueología y Etnología. Guatemala City: Ministerio de Cultura y Deportes, Instituto de Antropología e Historia y Asociación Tikal. Ciudad de Guatemala.

- Saturno, William, (2002). Archaeological Investigation and Conservation at San Bartolo, Guatemala. Foundation for the Advancement of Mesoamerican Studies (www.famsi.org).

- Saturno, William, (2003a). Sistine Chapel of the early Maya. National Geographic 204(6):72-77. Washington, DC.

- Saturno, William, (2003b). Proyecto Arqueológico Regional San Bartolo: resultados de la primera temporada de campo 2002. En Simposio de Investigaciones Arqueológicas en Guatemala, pp. 319-324 Instituto de Antropología e Historia; Asociación Tikal. Ciudad de Guatemala.

- Schele, Linda, (1986). The Founders of Lineages at Copán and Other Sites. Copán Note 8. Copán Mosaics Project y el Instituto Hondureño de Antropología e Historia. Copán, Honduras y el Departamento de Arte e Historia del Arte, University of Texas at Austin.

- Sharer, Robert, (1997). The Foundation of the Ruling Dynasty at Copán, Honduras: The Early Acropolis and Mesoamerican Interaction. Trabajo presentado en el Simposio A Tale of Two Cities: Copán and Teotihuacán. Departmento de Antropología, Harvard University, May 2-4, 1997. Cambridge.

- $\quad$ Sharer, Robert, W. Fash, L. Traxler, C. Carrelli, E. Bell, y C. Wells, (1999).

Early Classic Architecture Beneath the Copán Acropolis: A Research Update. Ancient Mesoamerica 10:3-23.

- Sharer, Robert, y D. Sedat, (1994). The Xukpi Stone: A Newly Discovered Early Classic Inscription from the Copán Acropolis. Part I: The Archaeology. Copán Note 113. Copán Mosaics Project y el Instituto Hondureño de Antropología e Historia. Copán, Honduras y el Departmento de Art e Historia del Arte, University of Texas at Austin.

- Sharer, Robert, L. Traxler, D. Sedat, E. Bell, M. Canuto y C. Powell, (1999). Early Classic Architecture beneath the Copán Acropolis. Ancient Mesoamerica 10:3-23. 
- Stuart, David, (1987). Ten Phonetic Syllables. Research Reports on Ancient Maya Writing 14. Center for Maya Research, Washington, D.C.

- Stuart, David, (2000). The Arrival of Strangers: Teotihuacán and Tollan in Classic Maya History. En Mesoamerica's Classic Hertiage, editado por D. Carrasco, L. Jones y S. Sessions, pp. 465-513. Boulder; Westview Press.

- Stuart, David, y L. Schele, (1986). Yax-K'uk-Mo', the Founder of the Lineage of Copán. Copán Note 6. Copán Mosaics Project y el Instituto Hondureño de Antropología e Historia. Copán, Honduras y el Departamento de Arte e Historia del Arte, University of Texas at Austin.

- Taube, Karl, (1989). The Maize Tamale, wah, in Classic Maya Epigraphy and Art. American Antiquity 54(1):31-51.

- Tedlock, Dennis, (1996). Popol Vuh. The Definitive Edition of the Mayan Book of the Dawn of Life and the Glories of Gods and Kings. New York: Simon y Schuster.

- Tozzer, Alfred M., (1941). Landa's Relación de las Cosas de Yucatán. Papers of the Peabody Museum of American Archaeology and Ethnology, no. 18. Cambridge, MA: The Peabody Museum, Harvard University Press. 